\title{
Weikangning therapy in functional dyspepsia and the protective role of $\mathrm{Nrf2}$
}

\author{
YUJUAN CHANG ${ }^{1,2}$, WEI WEI $^{2}$, LI TONG $^{3}$, YANJUN LIU $^{2}$, AIMIN ZHOU $^{4}$, \\ JIANDE CHEN $^{5}$, RUHAN WEI ${ }^{4}$, PING ZHANG ${ }^{6}$ and XIAOLAN SU ${ }^{2}$

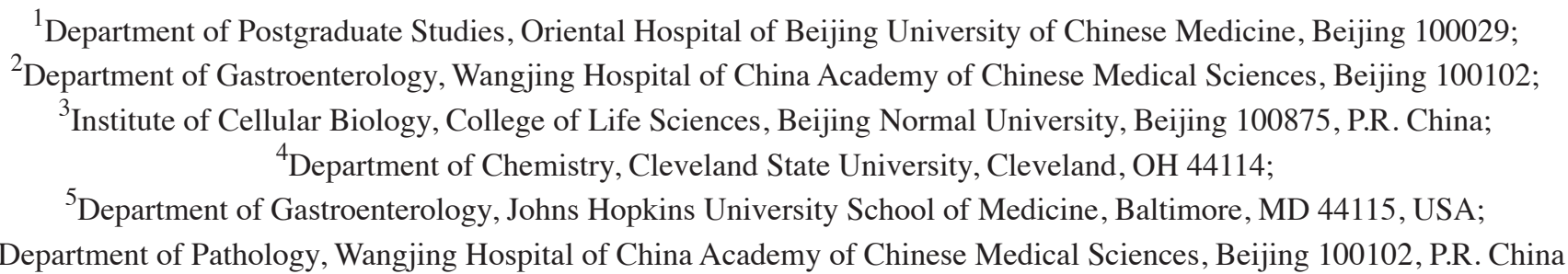

Received August 29, 2016; Accepted May 16, 2017

DOI: 10.3892/etm.2017.4892

\begin{abstract}
Functional dyspepsia (FD) is a non-organic gastrointestinal disorder that has a marked negative impact on quality of life. Compared with conventional pharmacological therapies, the traditional Chinese medicine weikangning (WKN) is a safe and effective treatment for FD. The present study aimed to determine the molecular mechanisms underlying the efficacy of WKN. The effect of different concentrations of WKN on the proliferation of the human gastric mucosal epithelial cell line GES-1 was assessed. The optimal WKN concentration to promote cell proliferation was determined, and this concentration was used to examine the effect of WKN compared with a domperidone-treated positive control group on the antioxidant capacity of GES-1 cells. The effect of WKN treatment on the growth and antioxidant activity of GES-1 cells was also assessed following nuclear factor erythroid 2 like 2 (Nrf2) knockdown. The optimal WKN dose for promoting cell growth was determined to be $0.025 \mathrm{mg} / \mathrm{ml}$; at this concentration the expression of the antioxidant proteins glutathione S-transferase $\mathrm{P}$ and superoxide dismutase 2 (SOD2) were significantly elevated $(\mathrm{P}<0.0001)$. Furthermore, the amount of reduced glutathione and activity of SOD2 were significantly increased $(\mathrm{P}<0.0001$ and $\mathrm{P}<0.01$, respectively), and malondialdehyde content was significantly decreased, compared with the controls $(\mathrm{P}<0.001)$. With WKN treatment, the transcription
\end{abstract}

Correspondence to: Dr Wei Wei, Department of Gastroenterology, Wangjing Hospital of China Academy of Chinese Medical Sciences, 6 Central South Road, Wangjing, Chaoyang, Beijing 100102, P.R. China

E-mail: changyujuanyi@163.com

Key words: functional dyspepsia, traditional Chinese medicine, weikangning, gastric mucosal epithelial cell, antioxidant capacity, nuclear factor erythroid 2 like 2 of Nrf2 and its downstream genes were significantly upregulated $(\mathrm{P}<0.01)$, and the level and nuclear distribution of Nrf2 protein was also markedly increased. Following Nrf2 silencing, the protective antioxidant effects of WKN treatment were impaired and GES-1 cell proliferation decreased. The results of the present study suggest that the efficacy of WKN in protecting gastric mucosal epithelial cells in FD is antioxidant-dependent and mediated by Nrf2 activation.

\section{Introduction}

Functional dyspepsia (FD) is a non-organic gastrointestinal (GI) disorder with persistent or recurrent sensations of early satiety, post-prandial fullness, nausea, and pain or discomfort in the upper abdomen $(1,2)$. According to recent statistics, FD affects 5-11\% of the population worldwide (3). Although FD is not a life-threatening disease, it necessitates numerous medical treatments and places a significant economic burden on patients (4). Furthermore, it has been reported that FD may develop into peptic ulceration (5).

Uncovering the pathogenesis of FD may contribute to the development of successful intervention strategies, which may in turn prevent symptomatic exacerbations of the disease and potentially even restore the digestive system to normal. There are a number of theories regarding the potential causes of FD, including genetic factors, infection with Helicobacter pylori, neurologic-hormonal disorders, autonomic disorders, stress and mental disorders, visceral hypersensitivity, and altered duodenal sensitivity to acids and lipids (1).

Several studies have explored the potential causes of FD, and efforts have been made to identify effective treatments; however, the optimal therapy for FD is yet to be determined (6). The majority of pharmacologic treatments that are currently available for FD have demonstrated limited efficacy (7). Several 5-hydroxytryptamine agonists, including tegaserod, cisapride, mosapride, pukabili, and renzapride, have demonstrated excellent efficacy in the treatment of FD via regulating GI dynamics; however, their safety has not been 
adequately demonstrated (8). For example, evidence indicates that tegaserod may increase the risk of myocardial infarction, sudden cardiac death and other cardiovascular events $(9,10)$. By contrast, cisapride may lead to QT interval prolongation, ventricular tachycardia and cardiac arrest (11).

The traditional Chinese medicine (TCM) weikangning (WKN) is a representative formula based on the classical TCM theory of 'Xi Kai Ku Jiang' (12). Several studies from the past decade have reported that WKN is a safe and effective treatment for FD $(12,13)$. The composition of WKN includes the following nine Chinese herbs: Radix Scutellariae, Rhizoma Zingiberis, Radix Codonopsis pilosulae, Rheum rhabarbarum, Radix Bupleuri, Radix Curcumae, Magnolia officinalis bark, Radix Paeoniae alba, and Rhizoma Corydalis (12). Pharmacological studies have indicated that Pinellia spp., Radix Codonopsis pilosulae, Villosum spp., Magnolia officinalis and Rheum rhabarbarum may strengthen GI smooth muscle tension (14-19). Furthermore, Scutellaria baicalensis and Coptis chinensis have been reported to function as anti-inflammatory and antiallergic mediators, and serve a dual-directional role in the regulation of GI smooth muscle, which may provide a pharmacological basis for WKN as a treatment for FD (20-22).

To learn more about the detailed molecular mechanism underlying the efficacy of WKN in the treatment of FD, our group previously conducted a proteomic study to identify the differences in GI protein expression in an FD rat model with and without WKN treatment (12). The results revealed that the protein expression levels of glutathione-S-transferase $\mathrm{P}$ (GSTP1) and superoxide dismutase 2 (SOD2) were decreased in the disease model group compared with the normal control group. However, following the administration of WKN, the expression of GSTP1 and SOD2 was enhanced.

According to previous studies, GSTP1 and SOD2 serve an important role in combating oxidative stress $(23,24)$. For example, Wang et al (23) demonstrated that upregulating the expression of GSTP1 and SOD2 significantly decreased oxidative stress by reducing reactive oxygen species (ROS) formation. In addition, a study by Dato et al (24) reported that genes belonging to pro-antioxidant signaling pathways, including GSTP1 and SOD2, were positively associated with longevity and functional status in very old age. Based on such observations and preliminary work by our group, it was hypothesized that oxidative stress may be an important component of the pathological mechanism of FD and that the efficacy of WKN may be due to reduced levels of oxidative stress. The results of these previous studies suggest that WKN may protect human GI cells by increasing antioxidant capacity, thereby maintaining the survival and growth of these cells and promoting recovery from FD. In addition, a previous study revealed that Nrf2 pathway played a critical role in antioxidation, anti-inflammation and protecting liver from oxidative damages in dyspepsia (25). Based on these results, it was investigated in the current study whether the antioxidant regulation of Nrf2, GSTP1 and SOD2 was involved underlying the WKN treatment for FD. Overall, the present study aimed to provide a theoretical basis for the use of TCMs in the treatment and prevention of FD, and to provide experimental data to support the hypothesis that oxidative injury is important in the development and exacerbation of FD.

\section{Materials and methods}

Cell culture. The human gastric mucosal epithelial cell line GES-1 was purchased from the cell bank of Culture Preservation Committee, Chinese Academy of Sciences (Beijing, China). GES-1 cells were cultured in RPMI-1640 medium (Gibco; Thermo Fisher Scientific, Inc., Waltham, MA, USA) containing $10 \%$ heat inactivated fetal bovine serum (Zhejiang Tianhang Biological Technology Co., Ltd., Zhejiang, China), 100,000 U/1 penicillin and $100 \mathrm{mg} / 1$ streptomycin in an atmosphere containing $5 \% \mathrm{CO}_{2}$ at $37^{\circ} \mathrm{C}$.

Establishment of the lentivirus-mediated nuclear factor erythroid 2 like 2 (Nrf2) gene-knockdown stable strain of GES-1 cells. Four pairs of short hairpin (sh)RNA species targeting Nrf2 mRNA were designed by Origene Technologies (Rockville, MD, USA; Table I) and cloned into Lenti-KD vectors (Pre-1102; Beijing Pregene Science and Technology Co., Ltd., Beijing, China) with blank vectors as controls. Using Lipofectamine ${ }^{\circledR}$ 2,000 Transfection reagent (Thermo Fisher Scientific, Inc.), these plasmids, along with the pMD2.0G and psPAX2 plasmids (both Addgene, Cambridge, MA, USA), were successively transfected into $293 \mathrm{~T}$ cells (ATCC, Manassas, VA, USA) to form a virus package. After each type of transfected $293 \mathrm{~T}$ cells were cultured in $5 \% \mathrm{CO}_{2}$ at $37^{\circ} \mathrm{C}$ for 3 days, a $2 \mathrm{ml}$ culture of supernatant-containing lentiviral particles was harvested by centrifugation at a speed of $300 \mathrm{x} \mathrm{g}$ at $4^{\circ} \mathrm{C}$ for $5 \mathrm{~min}$, and stored at $-80^{\circ} \mathrm{C}$ until required.

A total of $2 \times 10^{5}$ GES-1 cells were seeded into a 6 -well plate. Following overnight culture, the adherent cells were transfected at room temperature with the lentivirus-containing culture medium overnight, and cultured at $30^{\circ} \mathrm{C}$ in a $5 \% \mathrm{CO}_{2}$ incubator. The following day, the medium was replaced. At 48-72 $\mathrm{h}$ following transfection, $3 \mu \mathrm{g} / \mathrm{ml}$ puromycin was added for selection and the cells were sub-cultured in a $10 \mathrm{~cm}^{2}$ plate, with continued puromycin treatment. The Nrf2 expression of transduced cells was identified using western blotting as described below.

The gene knockdown mediated by the lentivirus with the shNrf2-3 sequence appeared to be the most effective (Fig. 1). The GES-1 cell strain transfected with this lentivirus was cryopreserved at $-80^{\circ} \mathrm{C}$ as the Nrf2 gene-knockdown stable strain, which is referred to as shNrf2 throughout the present study. At the same time, the GES-1 cells transfected with lentivirus containing shMock sequence (CCTAAGGTTAAGTCGCCC TCG) were cryopreserved and used as a control (shMock). After recovery, the culture conditions for the stable cell lines were the same as those described for the GES-1 cells above.

Drug preparation. WKN was prepared using the following ingredients: Rhizome Pinelliae Preparata, 9 g; Rhizoma Coptidis, 5 g; Radix Scutellariae, 9 g; Fructus Amomi villosi, 6 g; Rhizoma Zingiberis, 9 g; Radix C. pilosulae, 15 g; Radix Curcumae, $9 \mathrm{~g}$; M. officinalis bark, $9 \mathrm{~g}$; Rhizoma Corydalis vinegar, 10 g; Radix Bupleuri, 9 g; rhubarb, 6 g; fried almond, $9 \mathrm{~g}$; inflammatory licorice, $9 \mathrm{~g}$; Radix Paeoniae Alba, $15 \mathrm{~g}$; and Fructus Ziziphi jujubae, 4 g. All components were supplied by the Department of Pharmacy of Wangjing Hospital of China Academy of Chinese Medical Sciences (Beijing, China). WKN was decocted according to traditional methods. The WKN was 
Table I. Sequences of shRNAs targeting Nrf2 mRNA.

\begin{tabular}{ll}
\hline \multicolumn{1}{l}{ Oligo } & \multicolumn{2}{c}{ Sequence } \\
\hline shNrf2-1 & F:5'-GATCCGCACCTTATATCTCGAAGTTCTTCCTGTCAGAAAACTTCGAGATATAAGGTGCTTTTTG-3' \\
& R:5'-AATTCAAAAAGCACCTTATATCTCGAAGTTTCTGACAGGAAGAAACTTCGAGATATAAGGTGCG-3' \\
shNrf2-2 & F:5'-GATCCCCGGCATTTCACTAAACACAACTTCCTGTCAGATTGTGTTTAGTGAAATGCCGGTTTTTG-3' \\
& R:5'-AATTCAAAAACCGGCATTTCACTAAACACAATCTGACAGGAAGTTGTGTTTAGTGAAATGCCGGG-3' \\
shNrf2-3 & F:5'-GATCCCCCTGTTGATTTAGACGGTATCTTCCTGTCAGAATACCGTCTAAATCAACAGGGTTTTT-3' \\
& R:5'-AATTCAAAAACCCTGTTGATTTAGACGGTATTCTGACAGGAAGATACCGTCTAAATCAACAGGGG-3' \\
shNrf2-4 & F:5'-GATCCGCAGCAAACAAGAGATGGCAACTTCCTGTCAGATTGCCATCTCTTGTTTGCTGCTTTTG-3' \\
& R:5'-AATTCAAAAAGCAGCAAACAAGAGATGGCAATCTGACAGGAAGTTGCCATCTCTTGTTGCTGCG-3'
\end{tabular}

shRNA, short hairpin RNA; Nrf2, nuclear factor erythroid 2 like 2; F, forward; R, reverse.

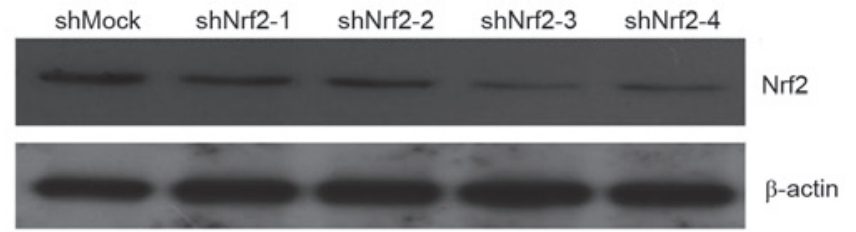

Figure 1. Expression of Nrf2 protein in GES-1 cell strains transfected with four types of shRNA or control shRNA. The expression of Nrf2 protein was assessed by western blotting. shRNA, short hairpin RNA; Nrf2, Nrf nuclear factor erythroid 2 like 2.

immersed in water for $30 \mathrm{~min}$, then $500 \mathrm{ml}$ water was added and decocted for $30 \mathrm{~min}$; the extract of $\sim 150 \mathrm{ml}$ was poured out, and another $500 \mathrm{ml}$ water was added followed by decocting again; finally the extract of the two steps were mixed, at a total volume of $300 \mathrm{ml}$. The solution was condensed to a volume of $400 \mathrm{ml}$. The $400 \mathrm{ml}$ crude extract solution was vacuum lyophilized for at least $3 \mathrm{~h}$ to obtain a powder using a freeze dryer (ALPHA1-4, Martin Christ GmbH, Osterode, Germany). The powder was stored until use at $-20^{\circ} \mathrm{C}$, and was dissolved in PBS to make the stock solution $(10 \mathrm{mg} / \mathrm{ml})$. The stock solution was sterilized using ${ }^{60} \mathrm{Co}-\gamma$ radiation (26) and centrifuged at $15,000 \times \mathrm{g}, 37^{\circ} \mathrm{C}$ for $20 \mathrm{~min}$ to remove the insoluble ingredients. The stock solution was stored at $-20^{\circ} \mathrm{C}$ and diluted to varying degrees depending on the concentration required for the different experimental procedures.

Domperidone (Sigma-Aldrich; Merck KGaA, Darmstadt, Germany) was dissolved in dimethyl sulfoxide (Sigma-Aldrich; Merck KGaA) to create a stock solution $(0.1 \mathrm{~mol} / \mathrm{l})$ and a working solution $(2.5 \mu \mathrm{mol} / \mathrm{ml})$, and was used as a positive control. PBS was used as the negative control. The drug treatment groups varied depending on the different experimental procedures, as outlined below.

\section{Proliferation activity assays}

Cell Counting Kit-8 (CCK-8) assay. A total of 1x10 4 GES-1 cells/well were seeded into a 96-well plate. The cells were then cultured in an atmosphere containing $5 \% \mathrm{CO}_{2}$ at $37^{\circ} \mathrm{C}$ for 4-6 h. Drug treatments were administered following adherence. Cells were divided into 10 groups and WKN was added at different concentrations $(0,0.025,0.05,0.1,0.2,0.3,0.4,0.5$, 0.6 and $0.7 \mathrm{mg} / \mathrm{ml})$. After 12,24 and $48 \mathrm{~h}$ of incubation at $37^{\circ} \mathrm{C}$,
$20 \mu \mathrm{l}$ CCK-8 solution (Beyotime Institute of Biotechnology, Haimen, China) was added to the wells. Cells were then cultured at $37^{\circ} \mathrm{C}$ for $1 \mathrm{~h}$. Optical density was measured at $450 \mathrm{~nm}$ using a microplate reader (POLARstar ${ }^{\circledR}$ Omega; BMG Labtech GmbH, Ortenburg, Germany). Four replicates were performed at each time-point for each group.

MTS assay. A total of $5 \times 10^{4}$ shMock or shNrf 2 cells/well were seeded into a 96 -well plate. The cells were then cultured with $5 \% \mathrm{CO}_{2}$ at $37^{\circ} \mathrm{C}$ for $4-6 \mathrm{~h}$. The drug treatment was performed following adherence. Cell culture media containing $0.025 \mathrm{mg} / \mathrm{ml} \mathrm{WKN}$ or equivalent amounts of PBS (as a blank control) was added to the wells. After 4, 48 and $96 \mathrm{~h}$ of incubation, $20 \mu \mathrm{l}$ MTS solution (Beijing Pregene Science and Technology Co., Ltd., Beijing, China) was added to the wells. Cells were then cultured at $37^{\circ} \mathrm{C}$ for $1 \mathrm{~h}$. Optical density was measured at $490 \mathrm{~nm}$ using the microlate reader. Six replicates were performed at each time point for each group.

Reverse transcription-quantitative polymerase chain reaction (RT-qPCR) analysis. A total of $1.5 \times 10^{6}$ cells/well were seeded into a 6 -well plate. The cells were cultured with $5 \%$ at $37^{\circ} \mathrm{C}$ for 4-6 h. The drug treatments (see the corresponding figure legend for the specific group settings and processing times) were performed following adherence. After treatment, total RNA was extracted using TRIzol reagent (Thermo Fisher Scientific, Inc.) and RT was performed using a Prime Script ${ }^{\mathrm{TM}}$ RT reagent kit (Takara Bio, Inc., Shiga, Japan) according to the manufacturer's protocol. PCR primers were synthesized by Shanghai Biotech Co., Ltd. (Shanghai, China) and are listed in Table II. The reaction was kept on ice in the dark until qPCR was performed and was comprised of the following components: $10 \mu 12 \mathrm{X}$ SYBR Premix Ex Taq ${ }^{\mathrm{TM}}$ (Takara Bio, Inc.), $0.5 \mu \mathrm{l}$ PCR forward primer $(10 \mu \mathrm{M}), 0.5 \mu \mathrm{l}$ PCR reverse primer $(10 \mu \mathrm{M}), 2 \mu \mathrm{l} \mathrm{cDNA}$ template and $7 \mu \mathrm{l}$ distilled $\mathrm{H}_{2} \mathrm{O}$. The thermocycling conditions were as follows: Initial denaturation at $95^{\circ} \mathrm{C}$ for $1 \mathrm{~min} ; 40$ cycles of $95^{\circ} \mathrm{C}$ for $15 \mathrm{sec}, 60^{\circ} \mathrm{C}$ for $15 \mathrm{sec}$ and $72^{\circ} \mathrm{C}$ for $45 \mathrm{sec}$; and a final extension at $72^{\circ} \mathrm{C}$ for $5 \mathrm{~min}$. Relative mRNA expression was calculated using the $2^{-\Delta \Delta C q}$ method (27). $\beta$-actin was used as the internal reference gene.

Western blotting. A total of $1.5 \times 10^{6}$ cells/well were seeded into a 6-well plate and cultured with $5 \% \mathrm{CO}_{2}$ at $37^{\circ} \mathrm{C}$ for $4-6 \mathrm{~h}$. 
Drug treatments (see the corresponding figure legend for the specific group settings and processing times) were administered following adherence. Total protein was extracted when cells were collected. After ultrasonic homogenization in the RIPA lysis buffer (150 $\mu \mathrm{l}$; Applygen Technologies, Inc., Beijing, China), cells were lysed at $4^{\circ} \mathrm{C}$ for another $10 \mathrm{~min}$, centrifuged $\left(15,000 \mathrm{x} \mathrm{g}, 4^{\circ} \mathrm{C}, 15 \mathrm{~min}\right)$, and the supernatant was transferred for analysis. Then protein was quantified using a BCA Protein Quantification kit (Beijing Biomed Biological Technology Co., Ltd., Beijing, China). Protein was denatured at $100^{\circ} \mathrm{C}$ for $5 \mathrm{~min}$, and $20-40 \mu \mathrm{g}$ of protein samples were loaded Next, SDS-PAGE was performed using the following settings: $4 \%$ stacking gel, $80 \mathrm{~V}$ for $30 \mathrm{~min}$; and a $12 \%$ resolving gel, $120 \mathrm{~V}$ for $1 \mathrm{~h}$. Proteins were then electrotransferred onto PVDF membranes. The membranes were blocked with 5\% nonfat milk in Tris-buffered saline $(\mathrm{pH} 7.5)$ with $0.1 \%$ Tween-20 for $1 \mathrm{~h}$ at room temperature. Primary antibodies directed against the following proteins were used: GSTP1 (P04906, Enzo Life Sciences, Farmingdale, NY, USA; 1:1,000), SOD2 (2299-1, Epitomics; Abcam, Cambridge, UK; 1:1,000), Nrf2 (ab62352, Abcam; 1:1,000), and $\beta$-actin (4970S, rabbit monoclonal antibody; Cell Signaling Technology, Danvers, MA, USA; 1:5,000). The membrane was incubated with the primary antibodies at $4^{\circ} \mathrm{C}$ overnight. The secondary horseradish peroxidase-conjugated immunoglobulin $\mathrm{G}$ antibody (ZB-2301; 1:3,000) was purchased from Zhongshan Jinqiao Biotechnology Co., Ltd. (Beijing, China). The membrane was incubated with the secondary antibodies at room temperature for $1 \mathrm{~h}$. Protein bands were visualized using an enhanced electrochemiluminescence western blot high-sensitivity detection kit (CW0049 M, Beijing Comwin Biotech Co., Ltd., Beijing, China) and images were captured using the Gel Doc ${ }^{\mathrm{TM}} \mathrm{XR}$ Gel Imaging system (Bio-Rad Laboratories, Inc., Hercules, CA, USA). Semi-quantitative analysis was performed using ImageJ 1.47 software (National Institutes of Health, Bethesda, MA, USA). The results are presented as percentages relative to the control groups.

Immunofluorescent staining. GES-1 cells were seeded into a culture dish with pretreated coverslips at a density of $10^{5}$ cells $/ 500 \mu \mathrm{l}$. After adherence, $0.025 \mathrm{mg} / \mathrm{ml} \mathrm{WKN}$ was added to the experimental groups, while an equivalent amount of PBS was added to the control group. After $24 \mathrm{~h}$ of treatment at $37^{\circ} \mathrm{C}$, the cells were harvested. The slides were soaked in PBS 3 times ( $3 \mathrm{~min} / \mathrm{soak}$ ) and fixed at room temperature for 15 min using $4 \%$ paraformaldehyde. Slides were subsequently soaked in PBS 3 times ( $3 \mathrm{~min} / \mathrm{soak}$ ) and penetrated with $0.5 \%$ Triton X-100 (in PBS) for $20 \mathrm{~min}$ at room temperature. Next, the slides were soaked in PBS and blotted dry. Subsequently, the slides were blocked with $6 \%$ normal goat serum (HyClone; GE Healthcare Life Sciences, Logan, UT, USA) in PBS for $20 \mathrm{~min}$ at room temperature. The blocking solution was removed and the slides were treated with primary antibodies directed against Nrf2 (ab137550, Abcam; 1:40) overnight at $4^{\circ} \mathrm{C}$. The slides were then rewarmed at $37^{\circ} \mathrm{C}$ for $45 \mathrm{~min}$, soaked in PBS 3 times ( $3 \mathrm{~min} / \mathrm{soak}$ ) and blotted dry. A fluorescent secondary antibody, goat anti-rabbit IgG H\&L Alexa Fluor ${ }^{\circledR} 647$ (ab150079; Abcam; 1:800), was added and the slides were incubated for $30 \mathrm{~min}$ at $37^{\circ} \mathrm{C}$. The slides were soaked in PBS 3 times ( $3 \mathrm{~min} / \mathrm{soak}$ ) and blotted dry.
Table II. Primers used in reverse transcription-quantitative polymerase chain reaction.

\begin{tabular}{ll}
$\begin{array}{l}\text { Target } \\
\text { gene }\end{array}$ & \multicolumn{1}{c}{ Sequence } \\
\hline GSTP1 & F: 5'-GGCTCTATGGGAAGGACCAGC-3' \\
& R: 5'-CACATAGTCATCCTTGCCCGCC-3' \\
SOD2 & F: 5'-TCAGCCCTAACGGTGGTGGA-3' \\
& R: 5'-AAGCCAACCCCAACCTGAGC-3' \\
Nrf2 & F: 5'-AGCATGCTACGTGATGAAGATGG-3' \\
& R: 5'-AGCTCAGAAAAGGTCAAATCCTCC-3' \\
NQO1 & F: 5'-CATCCCAACTGACAACCAGAT-3' \\
& R: 5'-CAGGGAAGCCTGGAAAGATAC-3' \\
HO-1 & F: 5'-ATGACACCAAGGACCAGAGC-3' \\
& R: 5'-GCATAAAGCCCTACAGCAACT-3' \\
GCLM & F: 5'-CACGAGGTCAGGAGTTTGAGA-3' \\
& R: 5'-AGCTGAGATTACAGGCGAGTG-3' \\
GCLC & F: 5'-CCCTCCTCCAAACTCAGACAT-3' \\
& R: 5'-TGGTGAGCAGTACCACAAACA-3' \\
$\beta$-actin & F: 5'-GCTGCCCTGAGGCACTCTTC-3' \\
& R: 5'-ATCCTGTCGGCAATGCCAGG-3'
\end{tabular}

GSTP1, glutathione-S-transferase P; SOD2, superoxide dismutase 2; Nrf2, nuclear factor erythroid 2 like 2; NQO1, NADPH dehydrogenase quninone 1; HO, heme oxygenase; GCLM, glutamate cystteine ligase modifier subunit; GCLC, glutamate cysteine ligase catalytic subunit; F, forward; $\mathrm{R}$, reverse.

The slides were treated with DAPI dye (Beyotime Institute of Biotechnology) at room temperature in the dark for $5 \mathrm{~min}$ to stain the cell nuclei. The slides were then washed with PBS 4 times ( $5 \mathrm{~min} /$ wash), sealed with mounting medium containing anti-fluorescence quenching reagent (Antifade Mounting Medium; Beyotime Institute of Biotechnology) and the edges were sealed with nail polish. The slides were observed under a fluorescence microscope and representative images were captured.

Detection of oxidative stress levels. Cells were lysed following drug treatment (see the corresponding figure legend for the specific group settings and processing times). The culture medium was removed and the cells were rinsed with pre-cooled PBS twice. Pre-cooled PBS $(1 \mathrm{ml})$ was added and cells were swept with a cell lifter, then transferred into a $1.5 \mathrm{ml}$ microcentrifuge tube. Centrifugation was performed at $4{ }^{\circ} \mathrm{C}$ and at $650 \mathrm{x} \mathrm{g}$ for $15 \mathrm{~min}$. The sediment was harvested and cytopreserved at $-80^{\circ} \mathrm{C}$. Different aspects of oxidative stress were detected using a reduced glutathione (GSH) content assay kit [intra-assay coefficient of variation (CV), 1.2\%; inter-assay CV: 3.86\%; sensitivity, 0.3-147.1 mg/1], a SOD enzyme activity assay kit (intra-assay $\mathrm{CV}, 5.05 \%$; inter-assay $\mathrm{CV}, 3.32 \%$; sensitivity, $0.5 \mathrm{U} / \mathrm{ml}$ ), and a maleic dialdehyde (MDA) content assay kit (intra-assay CV, 3.5\%; inter-assay CV, 4.11\%; sensitivity, $0.5-113 \mathrm{nmol} / \mathrm{ml}$ ). All kits were provided by Nanjing Jiancheng Bioengineering Institute (Nanjing, China). Cell lysis, the detection procedure and result calculations were all performed according to the manufacturer's protocol. 
Statistical analysis. All experiments were repeated $\geq 3$ times, and the results were analyzed using SPSS software (version 19.0; IBM Corp., Armonk, NY, USA). Data are represented as the mean \pm standard deviation. Student's t-test was used to analyze differences between two groups. $\mathrm{P}<0.05$ was considered to indicate a statistically significant difference.

\section{Results}

Low concentrations of WKN promote GES-1 cell proliferation. A CCK-8 assay was used to investigate cell proliferation and it was demonstrated that WKN at lower concentrations had the optimal effect in promoting the proliferation of GES-1 cells at all time points (Fig. 2). The pro-proliferative effects gradually weakened with an increase in the concentration of WKN. The optimal effect was observed at $0.025 \mathrm{mg} / \mathrm{ml}$.

Low concentrations of WKN increase the expression of GSTP1 and SOD2 in GES-1 cells. In our previous study of WKN treatment in FD model rats, proteomics were analyzed, and it was demonstrated that GSTP1 and SOD2 expression levels were decreased in the disease model group and upregulated following WKN treatment (12). It was therefore speculated that the growth-promoting effect of WKN treatment on GES-1 cells may be associated with the expression of GSTP1 and SOD2. Therefore, in the present study changes in GSTP1 and SOD2 expression following WKN treatment were investigated. Following treatment with WKN at different concentrations for $24 \mathrm{~h}$, the expression level of GSTP1 mRNA in GES-1 cells was significantly elevated compared with the control group $(\mathrm{P}<0.05$; Fig. 3A). However, no significant difference was observed between the domperidone treatment group and the control group (Fig. 3A). Although domperidone treatment significantly enhanced SOD2 mRNA levels ( $\mathrm{P}<0.05$; Fig. 3A), and GSTP1 and SOD2 protein levels $(\mathrm{P}<0.0001$ and $\mathrm{P}<0.001$, respectively; Fig. $3 \mathrm{~B})$, compared with the control group, $0.025 \mathrm{mg} / \mathrm{ml}$ WKN had a greater effect $(\mathrm{P}<0.0001$; Fig. 3B). As the concentration of WKN increased, its effect on GSTP1 and SOD2 mRNA and protein levels decreased (Fig. 3). These results further confirmed that the efficacy of WKN is concentration-dependent. When the concentration of WKN was $0.025 \mathrm{mg} / \mathrm{ml}$, the protective effect on GES-1 cells of promoting cell growth, and increasing GSTP1 and SOD2 expression levels, was the greatest. Therefore, this was chosen as the optimal concentration for subsequent experiments.

WKN decreases oxidative stress in GES-1 cells. GSTP1 and SOD2 serve important roles in combating oxidative stress. It was therefore hypothesized that the growth-promoting effect of WKN on gastric mucosal epithelial cells was associated with the regulation of oxidative stress. The GSH content, SOD activity and MDA content were measured as a proxy for the level of cellular oxidative stress (Fig. 4). When GES-1 cells were treated with $0.025 \mathrm{mg} / \mathrm{ml} \mathrm{WKN}, \mathrm{GSH}$ content and SOD activity increased significantly $(\mathrm{P}<0.0001$ and $\mathrm{P}<0.01$, respectively), while MDA content decreased significantly $(\mathrm{P}<0.001)$, compared with the control group. However, no significant changes in oxidative stress index levels were observed in the domperidone treatment group.

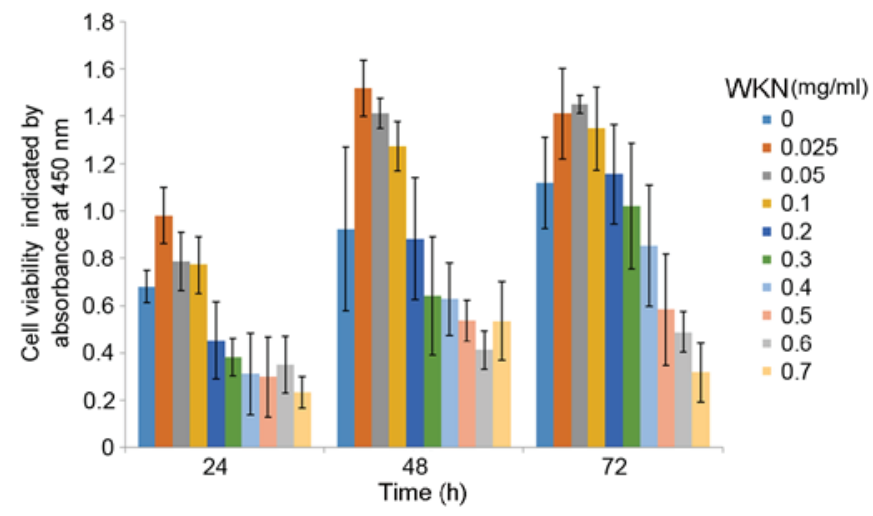

Figure 2. Proliferation GES-1 cells after 12, 24 or $48 \mathrm{~h}$ of WKN treatment at different concentrations $(0,0.025,0.05,0.1,0.2,0.3,0.4,0.5,0.6$ and $0.7 \mathrm{mg} / \mathrm{ml}$ ) detected using the Cell Counting Kit-8 assay. WKN, weikangning.

WKN increases the expression of Nrf2 and its target genes in GES-1 cells. A previous study identified gstpl and sod2 as two of the putative Nrf2-dependent genes (27) and Nrf2 has been identified as an important transcription factor that regulates oxidative stress (28-32). To determine whether WKN treatment enhanced the antioxidant capacity of cells via the promotion of Nrf2, GES-1 cells were treated with $0.025 \mathrm{mg} / \mathrm{ml}$ WKN and the expression level of the Nrf2 gene was measured as the processing time increased. The transcription level of Nrf2 was significantly upregulated following $2 \mathrm{~h}$ of WKN treatment $(\mathrm{P}<0.001$; Fig. 5A). This effect decreased with time. The transcription levels of four target genes of Nrf2 (NADPH dehydrogenase quninone 1 , heme oxygenase 1 , glutamate cysteine ligase modifier subunit and glutamate cysteine ligase catalytic subunit) were measured after $2 \mathrm{~h}$ (data not shown) and $4 \mathrm{~h}$ (Fig. 5B) of treatment with $0.025 \mathrm{mg} / \mathrm{ml} \mathrm{WKN}$. The results demonstrated that the transcription of the four genes increased significantly following $4 \mathrm{~h}$ of treatment $(\mathrm{P}<0.01$; Fig. 5B).

WKN increases GES-1 cell Nrf2 content and nuclear translocation. Under normal conditions, $\mathrm{Nrf} 2$ is anchored in the cytoplasm by Kelch-like ECH-associated protein 1 (Keap1). However, when cells are exposed to inducing stimuli, Nrf2 is activated, released from Keap1 and transported into the nucleus (33). Nrf2 then combines with the antioxidant response element (ARE) in the promoter region of downstream target genes, activating the transcription of target genes and transducing antioxidant signals (34). To clarify the impact of WKN treatment on Nrf2 transcriptional activity, changes in Nrf2 content and nuclear localization after WKN treatment were assessed in the present study. Immunofluorescence staining revealed that the Nrf 2 content of cells was markedly increased following WKN treatment and that Nrf2 protein was translocated into the nucleus (Fig. 5C).

Nrf2 silencing inhibits the protective effect of WKN on GES-1 cells. To further confirm that the growth-promoting effect of WKN on gastric mucosa epithelial cells was via the upregulation of Nrf2 and associated signaling pathways, a lentiviral-mediated Nrf2 gene knockdown GES-1 cell line was established. Western blotting revealed that Nrf2 protein expression decreased most markedly in shNrf22-3 silenced 

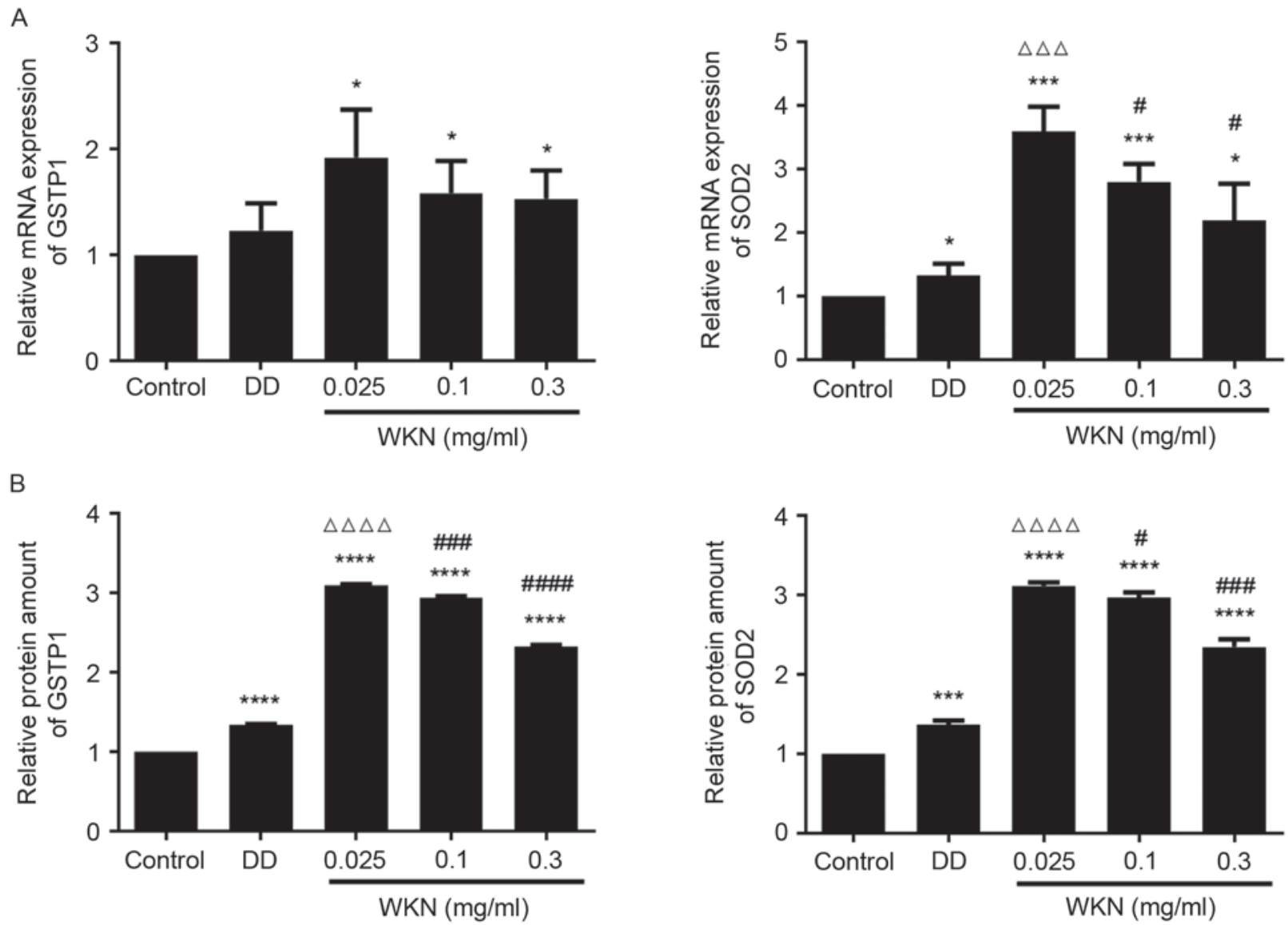

Figure 3. WKN treatment increases the expression of GSTP1 and SOD2 in GES-1 cells. GSTP1 and SOD2 (A) mRNA and (B) protein levels in GES-1 cells after $24 \mathrm{~h}$ treatment with $2.5 \mu \mathrm{mol} / \mathrm{ml}$ DD or WKN at. GES-1 cells treated with PBS were used as the control group and all results were normalized to the control. ${ }^{*} \mathrm{P}<0.05,{ }^{* * * *} \mathrm{P}<0.001$ and ${ }^{* * * * *} \mathrm{P}<0.0001$ vs. the control group. ${ }^{\Delta \Delta \Delta} \mathrm{P}<0.001$ and ${ }^{\Delta \Delta \Delta \Delta} \mathrm{P}<0.0001$ vs. the DD group. ${ }^{\#} \mathrm{P}<0.05,{ }^{\# \# \# "} \mathrm{P}<0.001$ and ${ }^{\# \# \# \#} \mathrm{P}<0.0001$ vs. the $0.025 \mathrm{mg} / \mathrm{ml}$ WKN group. WKN, weikangning; GSTP1, glutathione-S-transferase P; SOD2, superoxide dismutase 2; DD, domperidone.
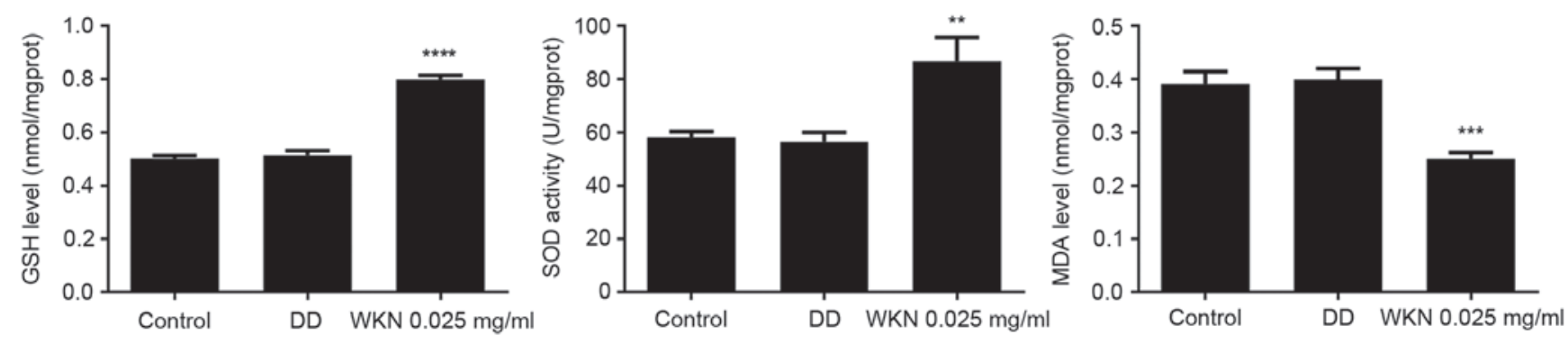

Figure 4. GSH content, SOD activity and MDA content of GES-1 cells following $24 \mathrm{~h}$ treatment with $2.5 \mu \mathrm{mol} / \mathrm{ml}$ DD or $0.025 \mathrm{mg} / \mathrm{ml}$ WKN. GES-1 cells treated with PBS solution were used as the control group. ${ }^{* *} \mathrm{P}<0.01$ and ${ }^{* * * *} \mathrm{P}<0.0001$ vs. the control group. GSH, glutathione; SOD, superoxide dismutase; MDA, maleic dialdehyde; WKN, weikangning; DD, domperidone.

cells compared with the shMock cells (Fig. 1). Thus, these shNrf cells were taken forward for further experiments.

In the shMock cells, treatment with $0.025 \mathrm{mg} / \mathrm{ml}$ WKN significantly increased the mRNA and protein expression of GSTP1 and SOD2 compared with the control group $(\mathrm{P}<0.05$; Fig. 6A and B). In shNrf2 cells, the mRNA and protein expression of GSTP1 and SOD2 were significantly lower compared with the shMock cells, before and after WKN treatment $(\mathrm{P}<0.01$; Fig. 6A and B). Furthermore, when Nrf2 was knocked down, the GSH content and SOD activity of GES-1 cells decreased significantly, and MDA increased significantly, compared with the shMock group $(\mathrm{P}<0.001$; Fig. 6C-E), despite treatment with WKN. These results indicate that the protective effect of WKN against cellular oxidative stress was significantly impaired by Nrf2 silencing.

The proliferative abilities of shNrf2 and shMock cells were examined using the MTS assay following 4, 48 and $96 \mathrm{~h}$ of treatment with $0.025 \mathrm{mg} / \mathrm{ml} \mathrm{WKN} \mathrm{(Fig.} \mathrm{6F).} \mathrm{This}$ demonstrated that $48 \mathrm{~h}$ of WKN treatment promoted the proliferation of shMock cells, but this effect decreased over time. Under Nrf2 silencing conditions, GES-1 cell growth was slower $(\mathrm{P}<0.0001$; control + shNrf2 vs. control + shMock group). Furthermore, with Nrf2 knockdown, the effect of $48 \mathrm{~h}$ WKN treatment cell 


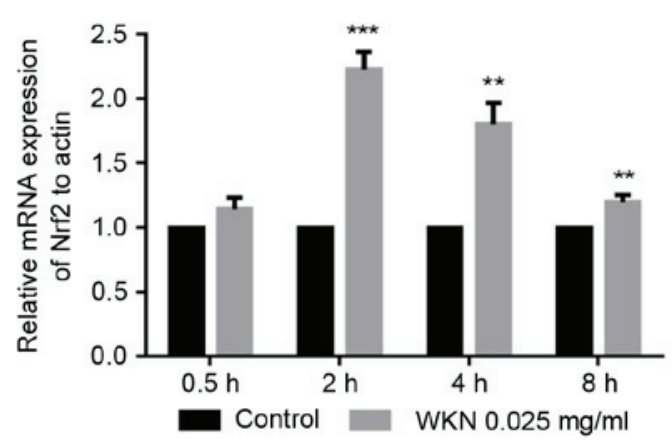

B

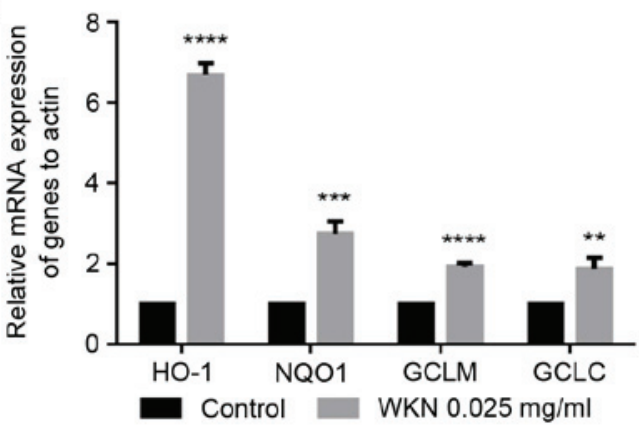

C
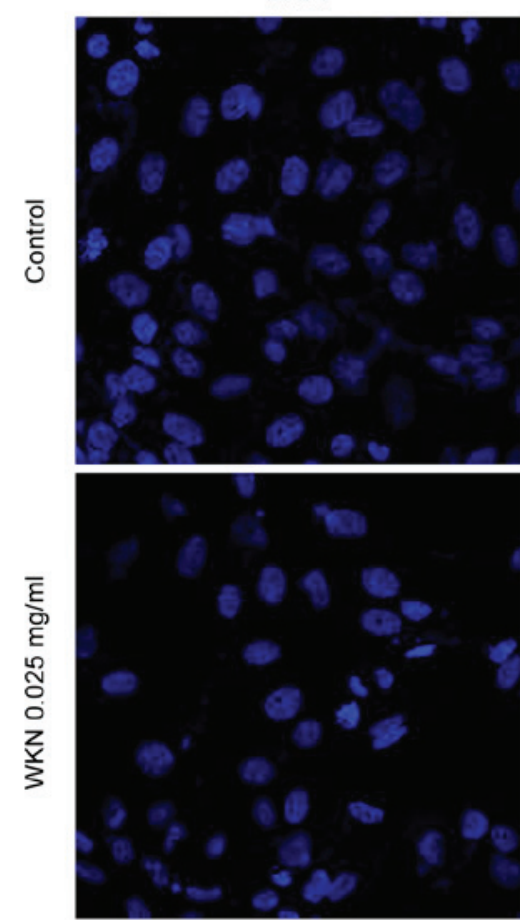

Nrf2- alexa fluor 647
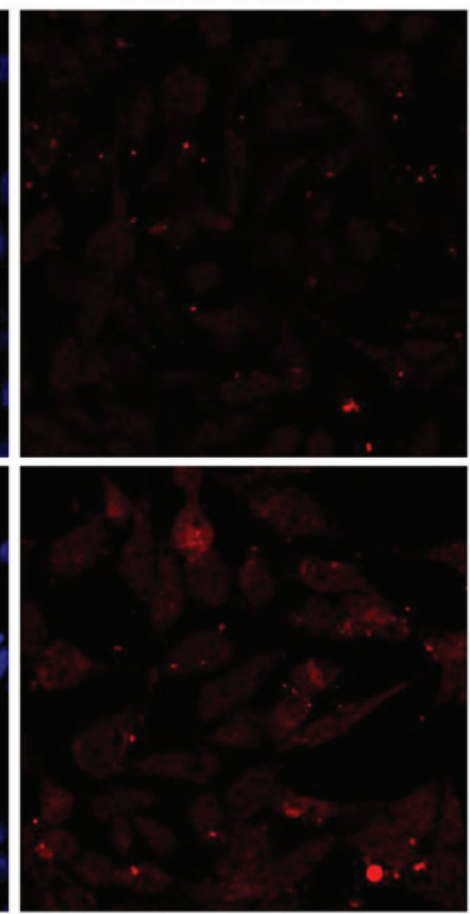
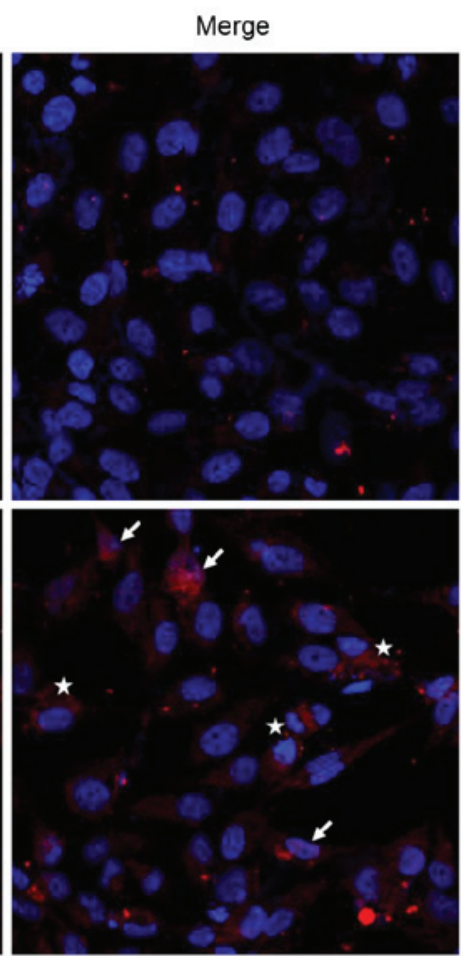

Figure 5. WKN treatment increases the transcription of Nrf2 in GES-1 cells. Transcription of (A) Nrf2 following $0.025 \mathrm{mg} / \mathrm{ml} \mathrm{WKN}$ treatment for $0.5,2,4$ and $8 \mathrm{~h}$, and (B) Nrf2 downstream target genes, GCLC, HO-1, GCLM and NQO1 in GES-1 cells following $0.025 \mathrm{mg} / \mathrm{ml} \mathrm{WKN} \mathrm{treatment} \mathrm{for} 4 \mathrm{~h}$. The results were normalized to the PBS control. (C) Representative immunofluorescence images of Nrf2 staining in GES-1 cells after $24 \mathrm{~h}$ treatment with $0.025 \mathrm{mg} / \mathrm{ml} \mathrm{WKN}$ or PBS solution. White stars indicate accumulated Nrf2 in the cytoplasm and white arrows indicate translocated Nrf2 in the nucleus. Magnification, x800. ${ }^{*} \mathrm{P}<0.05,{ }^{* * *} \mathrm{P}<0.01,{ }^{* * *} \mathrm{P}<0.001$ and ${ }^{* * * * *} \mathrm{P}<0.0001$ vs. the control group. WKN, weikangning; Nrf2, nuclear factor erythroid 2 like 2; GCLC, glutamate cysteine ligase catalytic subunit; HO-1, heme oxygenase 1; GCLM, glutamate cysteine ligase modifier subunit; NQO1, NADPH dehydrogenase quninone 1.

proliferation was significantly reduced $(\mathrm{P}<0.0001$ WKN + shNrf2 vs. WKN + shMock group).

\section{Discussion}

In a previous study, our group investigated the pathogenesis of FD, in addition to the therapeutic effect of WKN and the underlying molecular mechanism of this effect, from the perspective of proteomic analysis in a rat model of FD (12). It was demonstrated that the expression levels of GSTP1 and SOD2 were significantly upregulated in the WKN treatment group compared with the disease model group.

GSH-dependent and SOD enzymes are important cellular antioxidants that serve vital roles in resisting the damaging effects of external toxin and ROS $(35,36)$. GSTP1 is a member of the GSTP family, a subtype of GSTs. GSTP1 is capable of catalyzing the conjugate connections between GSH and charged complexes, so as to serve the function of detoxification. A previous study reported that different genotypes of the GSTP1 exon 5 are associated with intestinal metaplasia in the pre-gastric cancer stage, and positively interact with Helicobacter pylori infection in the intestinal metaplasia (37). SOD2 is primarily sub-cellularly located in the mitochondria and is an important component of the cellular antioxidant system (38-40). SOD2 maintains the balance between oxidation and antioxidation by reducing superoxides, including the superoxide anion (36). It has previously been reported that SOD2 knockout may be lethal in animals (41), whereas SOD2 overexpression significantly reduces lipid peroxidation, represses protein nitrosylation of protein tyrosine residues, inhibits cellular apoptosis and ameliorates ischemic tissue injury (42). In addition, SOD2 is able to inhibit the activation of the mitochondrial-dependent apoptotic signaling pathway by inhibiting the transfer of cytochrome $c$ from mitochondria to the cytoplasm (43-45). There is also evidence that SOD2 serves a more important role than SOD1 in cellular resistance to stress and injury (46). 

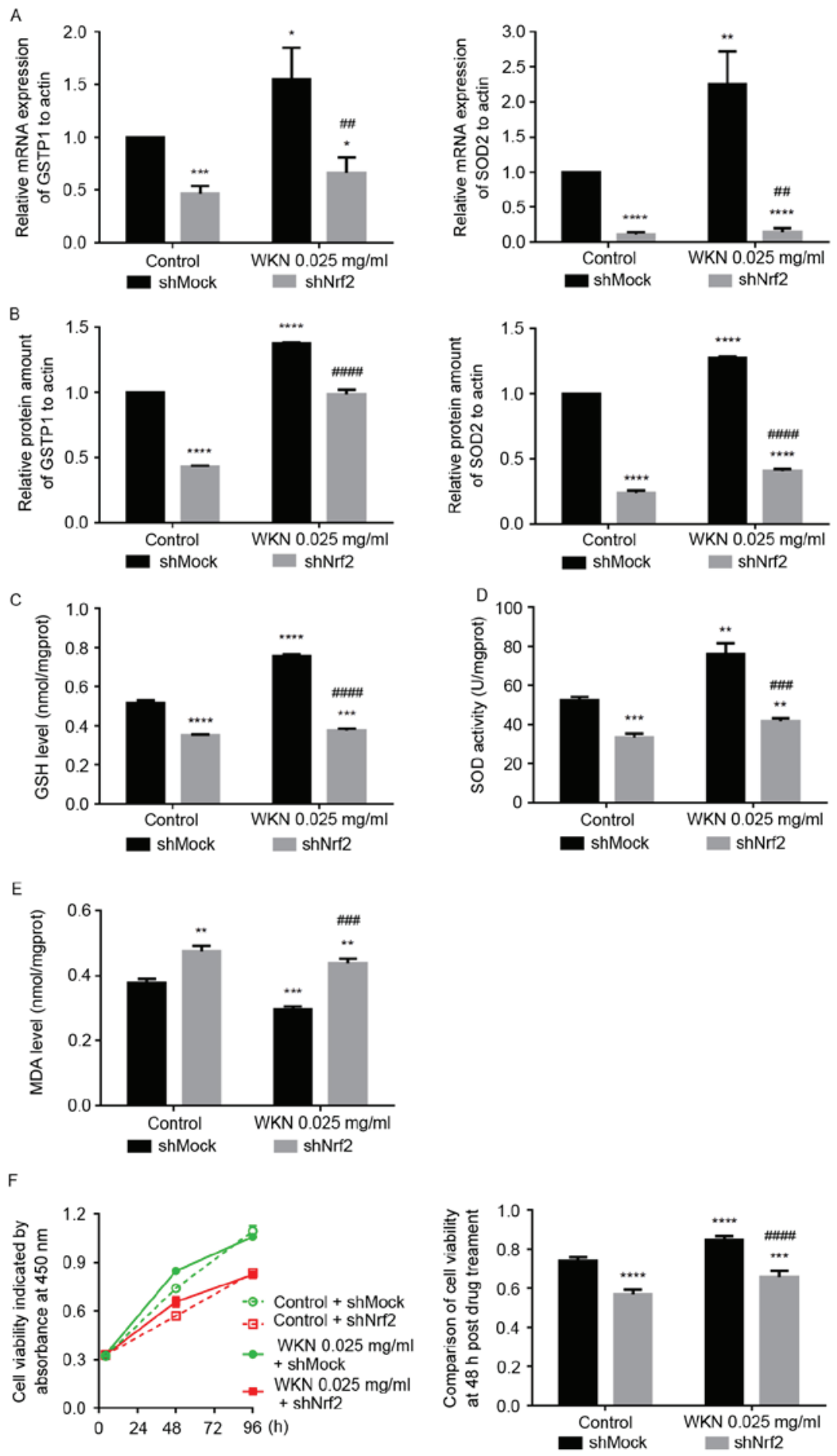

Figure 6. Nrf2 knockdown inhibits the protective effects of WKN on GES-1 cells. After $24 \mathrm{~h}$ treatment with $0.025 \mathrm{mg} / \mathrm{ml} \mathrm{WKN}$ or PBS solution, the levels of expression of GSTP1 and SOD2 (A) mRNA and (B) protein, (C) GSH content, (D) SOD activity and (E) MDA content in shMock cells and shNrf2 cells were asessed. Results were normalized to the values of the control + shMock group. (F) Effect of $0.025 \mathrm{mg} / \mathrm{ml}$ WKN treatment on the growth of GES-1 cells prior to and after Nrf2 knockdown was assessed using the MTT assay. ${ }^{*} \mathrm{P}<0.05,{ }^{* *} \mathrm{P}<0.01,{ }^{* * * *} \mathrm{P}<0.001$ and ${ }^{* * * * *} \mathrm{P}<0.0001$ vs. the control + shMock group. ${ }^{\# /} \mathrm{P}<0.01$,

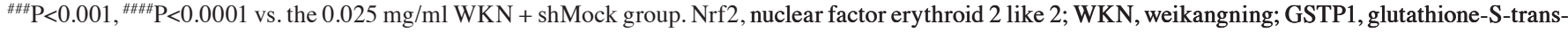
ferase P; SOD2, superoxide dismutase 2; GSH, glutathione; MDA, maleic dialdehyde; shRNA, short hairpin RNA.

Changes in the expression levels of GSTP1 and SOD2 in the FD rat model in our previous study suggested that oxidative stress may participate in the pathogenesis of FD (12). Previous studies have indicated that oxidative stress in GI tissues may serve an important role in the development of gastric damage and disorders, and the protective effects of certain pharmacological agents were reported to be associated with a reduction in oxidative stress (47-49). In the present study, SOD2 and GSTP1 were identified to be upregulated at the transcriptional and protein levels in human mucosal epithelial cells after WKN treatment. Furthermore, the GSH content, SOD activity and MDA content measured in the WKN-treated cells indicated that the WKN decreased oxidative stress in gastric mucosal epithelial cells. 
The results of the present study suggest that WKN promotes the expression and thus effects of antioxidant enzymes via the Nrf2/ARE signaling pathway, which was supported by the results of lentiviral-mediated Nrf2 knockdown. Nrf2 is an important transcription factor that exerts a protective effect against oxidative and chemical stress, through regulating the expression of antioxidant proteins via ARE interaction (35). The Nrf2-ARE signaling pathway is the main pathway that induces cell resistance to damage by exogenous substances and oxidative stress. Nrf2 also serves key roles in maintaining homeostasis of the cell microenvironment, and promoting cell growth and reproduction $(50,51)$. Nrf2 is able to protect the lungs, liver and digestive tract without cell or organ specificity $(52,53)$. Consequently, Nrf2 has great therapeutic potential for multi-organ protection $(53,54)$. The results of the present study demonstrated that Nrf2 may serve an essential role in GES-1 cells, as Nrf2 knockdown has an impact on growth and antioxidant activity. However, it remains to be elucidated whether the protective effect of WKN is entirely dependent on Nrf2 signaling.

In conclusion, combined with our previous research, the results of the present study suggest that the therapeutic efficacy of the TCM WKN as a treatment for FD relies on oxidative stress alleviation in GES-1 cells, which is mediated by Nrf2 signaling. The results of the present study also indicate indirectly that oxidative stress may be associated with the pathogenesis and development of GI disorders, something that has been proposed previously but is lacking in evidence $(55,56)$. The beneficial effects of TCMs on inflammatory GI diseases and associated tumorigenesis have been extensively studied, with certain reports suggesting that these treatments act via modulating the Nrf2 signaling pathway $(57,58)$. Future studies should test the results of the present study using an in vivo model of FD to increase the understanding of the pathology of functional GI disorders and potentially provide a clinical basis for WKN as a treatment for FD.

\section{Acknowledgements}

The present study was supported by the National Natural Science Fund (grant no. 81273746).

\section{References}

1. Brun R and Kuo B: Functional dyspepsia. Therap Adv Gastroenterol 3: 145-164, 2010.

2. Asano T, Aida S, Suemasu S, Tahara K, Tanaka K and Mizushima T: Aldioxa improves delayed gastric emptying and impaired gastric compliance, pathophysiologic mechanisms of functional dyspepsia. Sci Rep 5: 17519, 2015.

3. Talley NJ and Ford AC: Functional dyspepsia. N Engl J Med 374 896, 2016.

4. Lacy BE, Weiser KT, Kennedy AT, Crowell MD and Talley NJ: Functional dyspepsia: The economic impact to patients. Aliment Pharmacol Ther 38: 170-177, 2013.

5. El-Serag HB and Talley NJ: Systemic review: The prevalence and clinical course of functional dyspepsia. Aliment Pharmaco Ther 19: 643-654, 2004.

6. Zala AV, Walker MM and Talley NJ: Emerging drugs for functional dyspepsia. Expert Opin Emerg Drugs 20: 221-233, 2015.

7. Tack J, Bisschops R and Sarnelli G: Pathophysiology and treatment of functional dyspepsia. Gastroenterology 127: 1239-1255, 2004.

8. Lee M, Choi S and Im W: 5- $\mathrm{HT}_{4}$ Receptor agonists in the treatment of gastrointestinal motility disorders: Current status and perspective. Int J Gastroenterol Disord Ther 1: 108-116, 2014.
9. Loughlin J, Quinn S, Rivero E, Wong J, Huang J, Kralstein J, Earnest DL and Seeger JD: Tegaserod and the risk of cardiovascular ischemic events: An observational cohort study. J Cardiovasc Pharmacol Ther 15: 151-157, 2010.

10. Al-Judaibi B, Chande N and Gregor J: Safety and efficacy of tegaserod therapy in patients with irritable bowel syndrome or chronic constipation. Can J Clin Pharmacol 17: e194-e200, 2010.

11. Tack J, Camilleri M, Chang L, Chey WD, Galligan JJ, Lacy BE, Müller-Lissner S, Quigley EM, Schuurkes J, De Maeyer JH and Stanghellini V: Systematic review: Cardiovascular safety profile of 5-HT(4) agonists developed for gastrointestinal disorders. Aliment Pharmacol Ther 35: 745-767, 2012.

12. Wei W, Li X, Hao J, Zhang R, Guo J, Zong Y, Lu Y, Qu S and Tian J: Proteomic analysis of functional dyspepsia in stressed rats treated with traditional Chinese medicine 'Wei Kangning'. J Gastroenterol Hepatol 26: 1425-1433, 2011.

13. Wei W, Hao JJ and Tain J: Effect of Xinkai Kujiang method on gastric myoelectrical activity and autonomic nerve function of functional dyspepsia (FD) rat. Chin J Trad Chin Med and Pharm: 837-839, 2008

14. Wu H, Cai BC, Rong GX and Ye DJ: The effect of Pinellia processed by ginger juice on gastric and intestinal function of animals. Zhongguo Zhong Yao Za Zhi 19: 535-574, 1994 (In Chinese).

15. Park JW, Ryu B, Yeo I, Jerng UM, Han G, Oh S, Lee J and Kim J: Banha-sasim-tang as an herbal formula for the treatment of functional dyspepsia: A randomized, double-blind, placebo-controlled, two-center trial. Trials 11: 83, 2010.

16. Hou JY, Jiang ZW and He ZZ: Effects of Codonopsis pilosula (Franch) Nannf on gastric electricity, gastric motility and gastric emptying. Zhong Xi Yi Jie He Za Zhi 9: 31-32, 1989 (In Chinese).

17. Huang YL, Yen GC, Sheu F, Lin JY and Chau CF: Dose effects of the food spice cardamom on aspects of hamster gut physiology. Mol Nutr Food Res 51: 602-608, 2007.

18. Tian H, Huang D, Li T, Huang L, Zheng X, Tang D, Zhang L and Wang J: The protective effects of total phenols in magnolia officinalix rehd. Et wils on gastrointestinal tract dysmotility is mainly based on its influence on interstitial cells of cajal. Int $\mathbf{J}$ Clin Exp Med 8: 20279-20286, 2015.

19. Meng YB, Lei J, Hao ZM and Cao RL: Influence of rhubarb on gastrointestinal motility and intestinal mucosal barrier in patients with severe burn. Zhonghua Shao Shang Za Zhi 27: 337-340, 2011 (In Chinese).

20. Jung HS, Kim MH, Gwak NG, Im YS, Lee KY, Sohn Y, Choi H and Yang WM: Antiallergic effects of Scutellaria baicalensis on inflammation in vivo and in vitro. J Ethnopharmacol 141: 345-349, 2012.

21. Shin HS, Bae MJ, Choi DW and Shon DH: Skullcap (Scutellaria baicalensis) extract and its active compound, wogonin, inhibit ovalbumin-induced Th2-mediated response. Molecules 19: 2536-2545, 2014

22. Song SJ, Li FF, Xu Y, Zhang LH, Yuan F and Zhang Y: Effects of Honeysuckle flower and Scutellaria Baicalensis Georgi on constraction and electric activity of rabbit small intestine smooth muscle. Zhongguo Ying Yong Sheng Li Xue Za Zhi 25: 344-348, 2009 (In Chinese).

23. Wang Q, Chuikov S, Taitano S, Wu Q, Rastogi A, Tuck SJ, Corey JM, Lundy SK and Mao-Draayer Y: Dimethyl fumarate protects neural stem/progenitor cells and neurons from oxidative damage through Nrf2-ERK1/2 MAPK pathway. Int J Mol Sci 16: 13885-13907, 2015.

24. Dato S, Soerensen M, Lagani V, Montesanto A, Passarino G, Christensen K, Tan Q and Christiansen L: Contribution of genetic polymorphisms on functional status at very old age: A gene-based analysis of 38 genes ( 311 SNPs) in the oxidative stress pathway. Exp Gerontol 52: 23-29, 2014.

25. Zhang Q, Hu X, Hui F, Song Q, Cui C, Wang C and Zhao Q: Ethanol extract and its dichloromethane fraction of Alpinia oxyphylla Miquel exhibited hepatoprotective effects against CCl4-induced oxidative damage in vitro and in vivo with the involvement of Nrf2. Biomed Pharmacother 91: 812-822, 2017.

26. Fang XW and Wu JL: Feasibility of sterilizing traditional Chinese medicines by gamma-irradiation. Radiation Physics and Chemistry 52: 53-58, 1998.

27. Livak KJ and Schmittgen TD: Analysis of relative gene expression data using real-time quantitative PCR and the 2(-Delta Delta C(T)) method. Methods 25: 402-408, 2001. 
28. Wan Hasan WN, Kwak MK, Makpol S, Wan Ngah WZ and Mohd Yusof YA: Piper betle induces phase I \& II genes through Nrf2/ARE signaling pathway in mouse embryonic fibroblasts derived from wild type and Nrf2 knockout cells. BMC Complement Altern Med 14: 72, 2014

29. Nishinaka T, Ichijo Y, Ito M, Kimura M, Katsuyama M, Iwata K, Miura T, Terada T and Yabe-Nishimura C: Curcumin activates human glutathione S-transferase P1 expression through antioxidant response element. Toxicol Lett 170: 238-247, 2007.

30. Rubiolo JA, Mithieux G and Vega FV: Resveratrol protects primary rat hepatocytes against oxidative stress damage: Activation of the Nrf2 transcription factor and augmented activities of antioxidant enzymes. Eur J Pharmacol 591: 66-72, 2008.

31. Piao CS, Gao S, Lee GH, Kim DS, Park BH, Chae SW, Chae HJ and Kim SH: Sulforaphane protects ischemic injury of hearts through antioxidant pathway and mitochondrial K (ATP) channels. Pharmacol Res 61: 342-348, 2010.

32. Zhong JL, Edwards GP, Raval C, Li H and Tyrrell RM: The role of Nrf2 in ultraviolet A mediated heme oxygenase 1 induction in human skin fibroblasts. Photochem Photobiol Sci 9: 18-24, 2010.

33. Zipper LM and Mulcahy RT: The Keap1 BTB/POZ dimerization function is required to sequester Nrf2 in cytoplasm. J Biol Chem 277: 36544-36552, 2002.

34. Miller CJ, Gounder SS, Kannan S, Goutam K, Muthusamy VR, Firpo MA, Symons JD, Paine R 3rd, Hoidal JR and Rajasekaran NS: Disruption of Nrf2/ARE signaling impairs antioxidant mechanisms and promotes cell degradation pathways in aged skeletal muscle. Biochim Biophys Acta 1822: 1038-1050, 2012 .

35. Singh S: Cytoprotective and regulatory functions of glutathione $\mathrm{S}$-transferases in cancer cell proliferation and cell death. Cancer Chemother Pharmacol 75: 1-15, 2015.

36. Ramachandran A, Lebofsky M, Weinman SA and Jaeschke $\mathrm{H}$ : The impact of partial manganese superoxide dismutase (SOD2)-deficiency on mitochondrial oxidant stress, DNA fragmentation and liver injury during acetaminophen hepatotoxicity. Toxicol Appl Pharmacol 251: 226-233, 2011.

37. Sørensen M, Raaschou-Nielsen O, Brasch-Andersen C, Tjønneland A, Overvad $\mathrm{K}$ and Autrup $\mathrm{H}$ : Interactions between GSTM1, GSTT1 and GSTP1 polymorphisms and smoking and intake of fruit and vegetables in relation to lung cancer. Lung Cancer 55: 137-144, 2007.

38. Karnati S,Lüers G,Pfreimer S and Baumgart-VogtE: Mammalian SOD2 is exclusively located in mitochondria and not present in peroxisomes. Histochem Cell Biol 140: 105-117, 2013.

39. Lee YH, Boelsterli UA, Lin Q and Chung MC: Proteomics profiling of hepatic mitochondria in heterozygous Sod2+/- mice, an animal model of discreet mitochondrial oxidative stress. Proteomics 8: 555-568, 2008.

40. Zhang L, Chen CL, Kang PT, Jin Z and Chen YR: Differential protein acetylation assists import of excess SOD2 into mitochondria and mediates SOD2 aggregation associated with cardiac hypertrophy in the murine SOD2-tg heart. Free Radic Biol Med 108: 595-609, 2017.

41. Jang YC and Van Remmen H: The mitochondrial theory of aging: Insight from transgenic and knockout mouse models. Exp Gerontol 44: 256-260, 2009.

42. Perluigi M, Di Domenico F, Giorgi A, Schininà ME, Coccia R, Cini C, Bellia F, Cambria MT, Cornelius C, Butterfield DA and Calabrese V: Redox proteomics in aging rat brain: Involvement of mitochondrial reduced glutathione status and mitochondrial protein oxidation in the aging process. J Neurosci Res 88 3498-3507, 2010
43. Ong MM, Wang AS, Leow KY, Khoo YM and Boelsterli UA Nimesulide-induced hepatic mitochondrial injury in heterozygous Sod2 (+/-) mice. Free Radic Biol Med 40: 420-429, 2006.

44. Kasahara E, Lin LR, Ho YS and Reddy VN: SOD2 protects against oxidation-induced apoptosis in mouse retinal pigment epithelium: Implications for age-related macular degeneration. Invest Ophthalmol Vis Sci 46: 3426-3434, 2005.

45. Epperly MW, Sikora CA, DeFilippi SJ, Gretton JA, Zhan Q, Kufe DW and Greenberger JS: Manganese superoxide dismutase (SOD2) inhibits radiation-induced apoptosis by stabilization of the mitochondrial membrane. Radiat Res 157: 568-577, 2002

46. Fukui $M$ and Zhu BT: Mitochondrial superoxide dismutase SOD2, but not cytosolic SOD1, plays a critical role in protection against glutamate-induced oxidative stress and cell death in HT22 neuronal cells. Free Radic Biol Med 48: 821-30, 2010.

47. Zhao H, Zhu H, Lin Z, Lin G and Lv G: Compound 13, an a1-selective small molecule activator of AMPK, inhibits Helicobacter pylori-induced oxidative stresses and gastric epithelial cell apoptosis. Biochem Biophys Res Commun 463: $510-517,2015$.

48. Xie J, Lin Z, Xian Y, Kong S, Lai Z, Ip S, Chen H, Guo H, Su Z, Yang X, et al: (-)-Patchouli alcohol protects against Helicobacter pylori urease-induced apoptosis, oxidative stress and inflammatory response in human gastric epithelial cells. Int Immunopharmacol 35: 43-52, 2016.

49. Gezginci-Oktayoglu S, Orhan N and Bolkent S: Prostaglandin-E1 has a protective effect on renal ischemia/reperfusion-induced oxidative stress and inflammation mediated gastric damage in rats. Int Immunopharmacol 36: 142-150, 2016.

50. Zhou Y, Duan S, Zhou Y, Yu S, Wu J, Wu X, Zhao J and Zhao Y: Sulfiredoxin-1 attenuates oxidative stress via Nrf2/ARE pathway and 2-Cys Prdxs after oxygen-glucose deprivation in astrocytes. J Mol Neurosci 55: 941-950, 2015.

51. Mahmoud AM, Zaki AR, Hassan ME and Mostafa-Hedeab G: Commiphora molmol resin attenuates diethylnitrosamine/phenobarbital-induced hepatocarcinogenesis by modulating oxidative stress, inflammation, angiogenesis and Nrf2/ARE/HO-1 signaling. Chem Biol Interact 270: 41-50, 2017

52. Zhang DD: Mechanistic studies of the Nrf2-Keap1 signaling pathway. Drug Metab Rev 38: 769-789, 2006

53. Zhang H, Liu H, Davies KJ, Sioutas C, Finch CE, Morgan TE and Forman HJ: Nrf2-regulated phase II enzymes are induced by chronic ambient nanoparticle exposure in young mice with age-related impairments. Free Radic Biol Med 52: 2038-2046, 2012.

54. Niture SK, Kaspar JW, Shen J and Jaiswal AK: Nrf2 signaling and cell survival. Toxicol Appl Pharmacol 244: 37-42, 2010.

55. Turkkan E, Uslan I, Acarturk G, Topak N, Kahraman A, Dilek FH, Akcan Y, Karaman O, Colbay M and Yuksel S: Does Helicobacter pylori-induced inflammation of gastric mucosa determine the severity of symptoms in functional dyspepsia? J Gastroenterol 44: 66-70, 2009.

56. Suzuki H, Nishizawa T, Tsugawa H, Mogami S and Hibi T: Roles of oxidative stress in stomach disorders. J Clin Biochem Nutr 50: 35-39, 2012

57. Yang Y, Cai X, Yang J, Sun X, Hu C, Yan Z, Xu X, Lu W, Wang X and Cao P: Chemoprevention of dietary digitoflavone on colitis-associated colon tumorigenesis through inducing Nrf2 signaling pathway and inhibition of inflammation. Mol Cancer 13: 48,2014.

58. Havermann S, Chovolou Y, Humpf HU and Watjen W: Modulation of the Nrf2 signalling pathway in Hct116 colon carcinoma cells by baicalein and its methylated derivative negletein. Pharm Biol 54: 1491-1502, 2016. 